\title{
Outcome of Trimodal Therapy in Elderly Patients with Esophageal Cancer: Prognostic Value of the Charlson Comorbidity Index
}

\author{
DANIELE BERNARDI, EMANUELE ASTI, ALBERTO AIOLFI, \\ GIANLUCA BONITTA, ALBERTO LUPORINI and LUIGI BONAVINA \\ Division of General Surgery, Department of Biomedical Sciences for Health, \\ IRCCS Policlinico San Donato, University of Milan, Milan, Italy
}

\begin{abstract}
Background. In the real-world setting outside clinical trials, neoadjuvant chemoradiation therapy followed by esophagectomy may represent overtreatment in some elderly individuals with multiple comorbidities. Patients and Methods: Through an observational cohort study, consecutive patients with esophageal cancer treated with the protocol of the Chemoradiotherapy for Oesophageal Cancer Followed by Surgery Study (CROSS) were compared to patients of similar age who underwent upfront surgery during the same study period. Results: Fifty consecutive patients treated between 2010 and 2017 were enrolled. Thirty-two (64\%) were older than 65 years and three (9.4\%) were octogenarians. Eleven patients dropped-out of the protocol. Sixty-four patients undergoing upfront surgery served as controls. Compared to those who dropped out, patients who completed the protocol were younger $(p=0.002)$ and had a lower Charlson Comorbidity Index (CCI) ( $p=0.003)$; the CCI was also lower $(p=0.006)$ in the group treated with upfront surgery. The American Society of Anesthesiologists score did not discriminate between those who dropped out and patients who completed the protocol ( $p=0.178)$. Conclusion: Routine use of the CCI may help in the pretreatment risk stratification of elderly patients with esophageal carcinoma.
\end{abstract}

Esophageal cancer is the eighth most common cancer worldwide and the sixth cause of death due to cancer (1). It occurs predominantly in elderly people, with a peak incidence after 65 years of age (2). Elderly patients often

Correspondence to: Professor Luigi Bonavina, Piazza Edmondo Malan, 1, 20097 San Donato Milanese, Milan, Italy. Tel: +39 0252774840,e-mail: luigi.bonavina@unimi.it

Key Words: Esophageal cancer, elderly, neoadjuvant therapy, esophagectomy, comorbidity. have more preoperative comorbidities than younger patients. Since esophagectomy is still associated with high morbidity and mortality rates even at high-volume centers, surgeons are careful when making decisions for these patients (3) and a multidisciplinary assessment by a dedicated tumor board is nowadays standard of care at specialized centers (4).

Clinical trials have shown that neoadjuvant chemoradiation therapy followed by surgery can improve overall and diseasefree survival both in squamous cell and adenocarcinoma of the esophagus (5). The Chemoradiotherapy for Oesophageal Cancer Followed by Surgery Study (CROSS) regimen has recently become the most common neoadjuvant treatment modality for esophageal and esophagogastric junction carcinoma based on the assumption that a lower dose of radiotherapy $(41.4 \mathrm{~Gy})$ and concomitant carboplatin and paclitaxel chemotherapy may be safer and equally effective compared to previous protocols. However, in the real-world setting outside clinical trials, this strategy may represent overtreatment for some elderly individuals with multiple comorbidities (6).

The aim of this observational study was to investigate the clinical characteristics and outcomes of patients who were enrolled in the tri-modality regimen compared to those who underwent esophagectomy without previous chemoradiotherapy.

\section{Patients and Methods}

Patient sample and data collection. This retrospective cohort study was approved by the Institutional Review Board (PSD 0617). We used a prospectively constructed database to retrieve data for consecutive patients with carcinoma of the esophagus or the esophagogastric junction (Siewert type 1 or 2 ) treated with the CROSS protocol between July 2010 and March 2017.

Pre-treatment clinical staging included esophagogastroduodenoscopy with biopsies, computed tomography scan of the neck, chest and abdomen, endoscopic ultrasonography, positron-emission tomography and abdominal/neck ultrasound. Patients with locoregional disease [stage IB to III, seventh edition of the American Joint Committee on Cancer (AJCC) staging manual (7)] were considered for neoadjuvant chemoradiotherapy. Pre-treatment 
Eastern Cooperative Oncology Group Performance Status (ECOG PS), American Society of Anesthesiologists (ASA) score and ageadjusted Charlson Comorbidity Index $(\mathrm{CCI})(8,9)$ were recorded for all patients.

The neoadjuvant chemoradiotherapy regimen consisted of carboplatin and paclitaxel and concurrent radiotherapy with intensity-modulated radiotherapy-volumetric-modulated arc technology with single daily fraction of $1.8 \mathrm{~Gy}$, five days a week, for a total dose of 41.4-50.4 Gy. Patients were re-staged and clinically re-evaluated at the end of neoadjuvant therapy to confirm surgical indication. Surgery was performed 4 to 8 weeks after completion of neoadjuvant treatment.

The choice of operative approach was based on the patient's physiological status, histology, and tumor site. An Ivor-Lewis esophagectomy, either with hybrid or total minimally invasive approach, or a McKeown esophagectomy (prone thoracoscopy, laparoscopy and left cervicotomy) were performed (10). Upper abdominal and mediastinal lymphadenectomy and alimentary tract reconstruction with a gastric tube were performed in all cases. Pathological staging was carried out according to the TNM classification (7). Consecutive patients of similar age undergoing upfront esophagectomy during the same study period served as a control group.

Post-treatment complications and mortality. Chemoradiotherapyrelated toxicities were established according to the Common Terminology Criteria for Adverse Events (CTCAE v. 4.0, 2009) (11). Neoadjuvant treatment was stopped for grade III or more toxicity.

After surgery, all patients were admitted to the Intensive Care Unit for at least 24 hours. Oral intake of water usually started on postoperative day 3; solid food was allowed after a negative contrast swallow study on day 5 or 6 . Anastomotic leak was diagnosed with gastrography in swallow study, computed tomographic scan with oral contrast, with/without endoscopy. Pneumonia was defined as a clinically relevant radiological lung infiltrate necessitating antibiotic therapy. In-hospital and 90-day mortality were recorded.

Statistical analysis. Continuous data are presented as median and interquartile-range. Categorical variables are shown as frequency and percentages. The chi-square or Kruskal-Wallis test were performed as appropriate. Dunn's test (12) or Fisher's exact tests with Bonferroni correction were used for post-hoc tests in pairwise multiple comparisons. Confidence intervals for proportions at $95 \%$ confidence level were computed using normal approximation. Kaplan-Meier estimator for censored data was used to estimate overall survival probability, using log-rank test to compare survival curves. Two-sided $p$-values were computed, $p$ and $p$-value of 0.05 or less were considered statistically significant. All analyses were carried out with R 3.2.2 (13).

\section{Results}

Neoadjuvant therapy. Table I summarizes the demographic and clinical characteristics of the 50 patients submitted to the CROSS protocol. The median age was 66 years. Most patients were males (69.2\%) and smokers (62\%). The predominant histological subtype was squamous cell carcinoma $(70 \%)$ and the majority of patients had stage III
Table I. Baseline patient characteristics $(n=50)$.

\begin{tabular}{|c|c|}
\hline Characteristic & Value \\
\hline Median age (range), years & $66(38-93)$ \\
\hline \multicolumn{2}{|l|}{ Gender, n (\%) } \\
\hline Male & $36(69.2 \%)$ \\
\hline Female & $14(30.8 \%)$ \\
\hline Median BMI (range), kg/m² & $23.8(16-30)$ \\
\hline Median BWL (range), $\%$ & $7.75 \%(0.0-30.3 \%)$ \\
\hline Smoking history, $\mathrm{n}(\%)$ & $31(62 \%)$ \\
\hline Alcohol abuse (>3 UA/d) & $2(4 \%)$ \\
\hline GERD/Barrett's esophagus, n (\%) & $7(14 \%)$ \\
\hline Median CCI (IQR) & $6(2)$ \\
\hline \multicolumn{2}{|l|}{ ASA score, n (\%) } \\
\hline 1 & $4(8 \%)$ \\
\hline 2 & $34(68 \%)$ \\
\hline 3 & $12(24 \%)$ \\
\hline \multicolumn{2}{|l|}{ ECOG PS, n (\%) } \\
\hline 0 & $14(28 \%)$ \\
\hline 1 & $30(60 \%)$ \\
\hline 2 & $6(12 \%)$ \\
\hline \multicolumn{2}{|l|}{ Tumor site, n (\%) } \\
\hline Upper/middle & $19(38 \%)$ \\
\hline Distal/GEJ & $31(62 \%)$ \\
\hline \multicolumn{2}{|l|}{ Histology, n (\%) } \\
\hline SCC & $35(70 \%)$ \\
\hline $\mathrm{ADC}$ & $15(30 \%)$ \\
\hline \multicolumn{2}{|l|}{ Grading, n (\%) } \\
\hline $1-2$ & $29(58 \%)$ \\
\hline $3-4$ & $21(42 \%)$ \\
\hline \multicolumn{2}{|l|}{ Clinical T stage, n (\%) } \\
\hline 1 & $1(2 \%)$ \\
\hline 2 & $2(4 \%)$ \\
\hline 3 & $38(76 \%)$ \\
\hline 4 & $9(18 \%)$ \\
\hline \multicolumn{2}{|l|}{ Clinical N stage, $\mathrm{n}(\%)$} \\
\hline 0 & $2(4 \%)$ \\
\hline 1 & $37(74 \%)$ \\
\hline 2 & $11(22 \%)$ \\
\hline \multicolumn{2}{|l|}{ Pre-treatment stage, $\mathrm{n}(\%)^{*}$} \\
\hline I-II & $5(10 \%)$ \\
\hline III & $45(90 \%)$ \\
\hline
\end{tabular}

BMI: Body mass index; BWL: body weight loss; UA/d: units of alcohol/day; GERD: gastro-esophageal reflux disease; CCI: Charlson comorbidity index; IQR: interquartile range; ASA: American Society of Anesthesiologists; ECOG PS: Eastern Cooperative Oncology Group Performance Status; GEJ: gastro-esophageal junction; SCC: squamous cell carcinoma; ADC: adenocarcinoma. *According to the seventh edition of the AJCC staging manual (7).

disease (90\%) according to the seventh edition of the AJCC staging manual (7). The median CCI was 6 , ranging from 2 to 10 . All patients received carboplatin and paclitaxel (3 to 7 cycles) and radiotherapy (41.4 to $50.4 \mathrm{~Gy}$ ).

There was one case of death due to cardiac toxicity and seven grade III or greater adverse events (three hematological and four non-hematological) during therapy. 
Table II. Baseline characteristics of patients who dropped out and those who completed the neoadjuvant (CROSS protocol).

\begin{tabular}{lccc}
\hline Characteristic & $\begin{array}{c}\text { Dropped out } \\
\mathrm{n}=11\end{array}$ & $\begin{array}{c}\text { Completed } \\
\mathrm{n}=39\end{array}$ & $p$-Value \\
\hline Median age (IQR), years & $71(12)$ & $65(12)$ & 0.002 \\
Male, n (\%) & $9(82)$ & $27(69)$ & 0.659 \\
Median BMI (IQR), kg/m 2 & $24(6)$ & $24(4)$ & 0.535 \\
Median CCI (IQR) & $8(3)$ & $5(2)$ & 0.003 \\
SCC, n (\%) & $9(81.8 \%)$ & $26(66.7 \%)$ & 0.551 \\
Stage III, n (\%) & $11(100 \%)$ & $34(87.2 \%)$ & 0.495 \\
\hline
\end{tabular}

BMI: Body mass index; CCI: Charlson Comorbidity Index; IQR: interquartile range; SCC: squamous cell carcinoma.

Four patients dropped-out of the protocol because of pulmonary embolism $(n=3)$ or toxicity $(n=1)$. Two patients had a worsening of their ECOG PS from 2 to 3, and therefore were not considered fit for surgery. Disease progression to stage IV during chemoradiotherapy was documented in three patients. As shown in Table II, age and CCI were significantly higher in patients who dropped-out ( $p=0.002$ and $p=0.003$, respectively).

Overall, $11(22 \%)$ patients did not undergo esophagectomy after chemoradiotherapy. Of these, one patient who had clinical complete response and negative endoscopic biopsies refused surgery.

Comparison of neoadjuvant therapy and upfront esophagectomy. During the same study period, 140 consecutive patients with esophageal cancer underwent upfront esophagectomy. Sixty-four of these patients who were older than 65 years were compared with patients who dropped-out $(\mathrm{n}=11)$ or completed $(\mathrm{n}=21)$ the CROSS protocol (Table III).

There was no statistically significant difference between groups in term of age, sex, body mass index, preoperative serum albumin level, or disease stage. There was a statistically significant difference in the CCI score between patients who dropped out and patients who completed the protocol $(p=0.031)$, and between patients who dropped out the protocol and patients who underwent upfront surgery $(p=0.006)$, even without difference in age. There was a significantly higher prevalence of ASA score 3 among patients who dropped-out from the CROSS regimen. However, the ASA score did not discriminate between patients who dropped-out and those who completed the protocol $(p=0.178)$.

Surgical therapy. An Ivor-Lewis esophagectomy was performed in the majority $(64 \%)$ of patients. There were no statistically significant differences in postoperative morbidity, mortality, and $\mathrm{R} 1$ resection rates between elderly patients who completed the CROSS protocol and those who

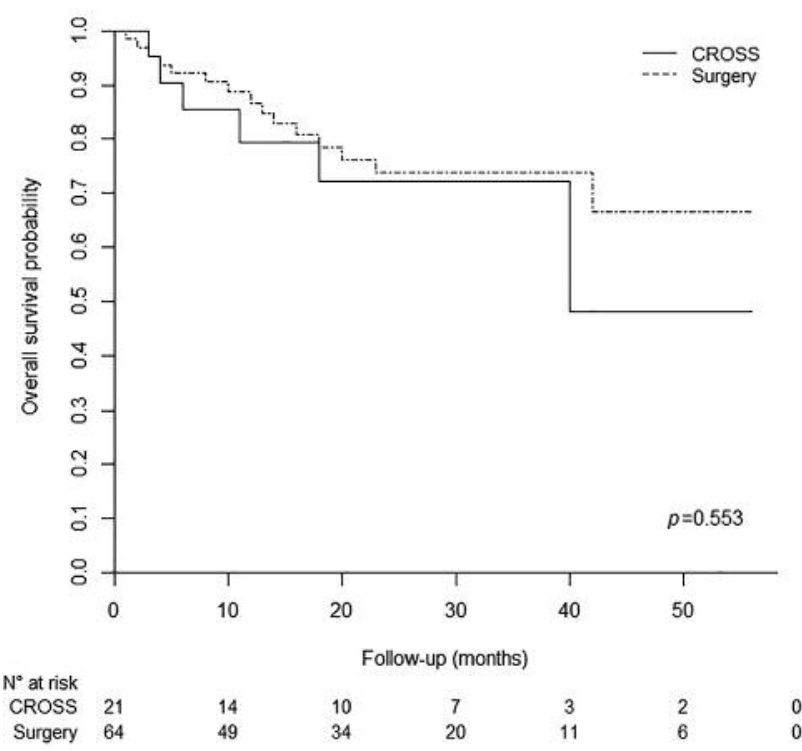

Figure 1. Comparison of overall survival between patients treated by trimodal therapy and those who underwent upfront surgery.

underwent upfront esophagectomy (Table III). Notably, all octogenarian patients who received neoadjuvant chemoradiation dropped-out of the protocol because of pulmonary embolism in two cases and worsening of performance status to grade 3 in one case. In addition, two (22\%) out of 10 octogenarian patients who underwent upfront esophagectomy had an anastomotic leak and died postoperatively.

The median follow-up was 20 months in patients who completed the CROSS protocol and 21.5 months in the those who underwent upfront surgery. There was no statistically significant difference in the overall survival probability between the two groups (log-rank test, $p=0.553$ ). At 40 months, the Kaplan-Meier estimate was 0.48 (95\% confidence interval $=0.21-1.0)$ in the neoadjuvant group and $0.73(95 \%$ confidence interval $=0.63-0.87)$ in the upfront surgery group (Figure 1).

\section{Discussion}

Most patients with esophageal cancer are older than 65 years and present with multiple comorbidities. Age, life expectancy, functional status, comorbidities, and quality of life have to be taken into account when planning a multimodal treatment. Given the under-representation of elderly patients in clinical trials, there are limited data regarding tolerance of trimodality treatments in this patient population (6). While CCI and ASA scores have already been associated with postoperative morbidity and mortality 


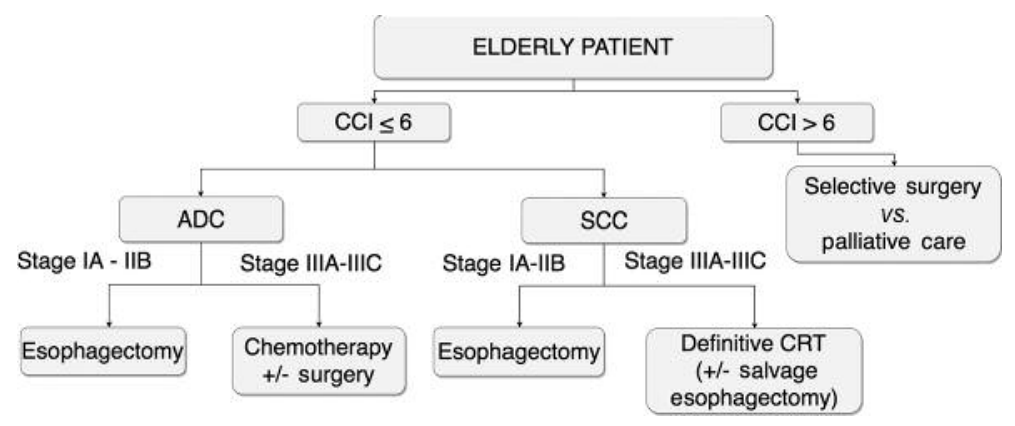

Figure 2. Proposed treatment algorithm for elderly patients. CCI: Age-adjusted Charlson Comorbidity Index; ADC: adenocarcinoma; SCC: squamous cell carcinoma; CRT: chemoradiotherapy.

Table III. Comparison of baseline characteristics and postoperative morbidity and mortality in elderly patients according to treatment.

\begin{tabular}{|c|c|c|c|c|}
\hline Characteristic & Dropped out $n=11$ & Completed $n=21$ & Upfront surgery $n=64$ & $p$-Value \\
\hline Median age (IQR), years (\%) & $71(12)$ & $68(9)$ & $71.5(9.5)$ & 0.200 \\
\hline Gender M, n (\%) & $9(82)$ & $13(62)$ & $52(81)$ & 0.173 \\
\hline Smoking history, n (\%) & $6(55)$ & $13(62)$ & $18(28)$ & $0.011^{\mathrm{a}}$ \\
\hline \multicolumn{5}{|l|}{ ASA, n $(\%)$} \\
\hline 1 & $0(0)$ & $0(0)$ & $5(8)$ & 0.268 \\
\hline 2 & $5(45)$ & $16(76)$ & $48(75)$ & 0.116 \\
\hline 3 & $6(55)$ & $5(24)$ & $11(17)$ & $0.024^{b}$ \\
\hline CCI (IQR) & $8.0(2.5)$ & $6.0(2.0)$ & $6.0(2.0)$ & $0.024^{\mathrm{c}}$ \\
\hline Median BMI (IQR), $\mathrm{kg} / \mathrm{m}^{2}$ & $24.2(5.9)$ & $24(5.2)$ & $24.6(5)$ & 0.384 \\
\hline Median serum albumin (IQR), g/dl & $3.8(0.4)$ & $4.1(0.5)$ & $4.0(0.5)$ & 0.354 \\
\hline $\mathrm{SCC}, \mathrm{n}(\%)$ & $9(82)$ & $14(67)$ & $12(19)$ & $<0.001^{\mathrm{d}}$ \\
\hline Stage III, n $(\%)$ & $11(100)$ & $20(95)$ & $58(91)$ & 0.478 \\
\hline Anastomotic leak, n (\%) & 0 & $4(19)$ & $4(6.3)$ & 0.189 \\
\hline Pneumonia, n (\%) & 0 & $1(4.8)$ & $2(3.1)$ & 0.999 \\
\hline $\mathrm{R} 1, \mathrm{n}(\%)$ & 0 & $2(9.5)$ & $8(12.5)$ & 0.999 \\
\hline Postoperative mortality, $\mathrm{n}(\%)^{*}$ & 0 & $2(9.5)$ & $4(6.3)$ & 0.986 \\
\hline Anastomotic insufficiency & 0 & 1 & 2 & \\
\hline ARDS & 0 & 1 & 0 & \\
\hline Hemothorax & 0 & 0 & 1 & \\
\hline Diease progression & 0 & 0 & 1 & \\
\hline
\end{tabular}

M: Male; IQR: interquartile range; ASA: American Society of Anesthesiologists; CCI: Charlson Comorbidity Index; BMI: body mass index; SCC: squamous cell carcinoma; R1: microscopic positive margin; ARDS: acute respiratory distress syndrome. *90-Day. aDropped out vs. completed: $p=0.999, v s$. surgery only: $p=0.474$; Completed $v s$. surgery only: $p=0.025$. bropped out $v s$. completed: $p=0.178, v s$. surgery only: $p=0.006$; Completed $v s$. surgery only: $p=0.720$. ${ }^{C}$ Dropped out $v s$. completed: $p=0.031$, vs. surgery only: $p=0.006$; Completed $v s$. surgery only: $p=0.741$. dDropped out $v s$. completed: $p=0.999, v s$. surgery only: $p<0.001$; Completed $v s$. surgery only: $p<0.001$.

(14), their relationship with tolerance to neoadjuvant chemoradiotherapy is unknown. The main finding of this study is that routine use of $\mathrm{CCI}(8,9)$ as a prognostic indicator may help in pretreatment risk stratification.

Preoperative chemoradiotherapy has become an accepted approach worldwide based on the findings of the CROSS trial $(15,16)$. However, the median patient age in the original study was 60 years, and this makes it difficult to generalize the study findings to an older patient population.
Previous studies have not shown significant differences in postoperative mortality rates between elderly and young patients undergoing neoadjuvant chemoradiation, but the morbidity rate was higher in older patients (17-20). However, none of these studies provide information about age and comorbidities of patients who dropped-out of the neoadjuvant protocol because of toxicity or other reasons. Interestingly, Ruol et al. found that patients who had grade III or IV toxicity during chemoradiation therapy or had to 
stop treatment due to toxicity, had significantly higher postoperative mortality compared to those who underwent surgery alone $(6.9 \%$ vs. $1.1 \%, p=0.026)$ (17). The FFCD 9901 trial also showed a higher rate of postoperative mortality in patients who underwent surgery alone $(11.1 \%$ vs. $3.4 \%$, respectively, $p=0.049$ ) (20). Klevebro et al. showed that there was no statistically significant difference in the incidence of postoperative complications, but the median Clavien-Dindo complication grade was significantly higher in the chemoradiation therapy group (22).

In our experience, 11 (22\%) out of 50 patients droppedout of the protocol during or at the end of chemoradiation therapy, mainly due to toxicity or worsening of their PS $(64 \%)$ or progression to stage IV disease $(27 \%)$. Their median age was 71 years and they had a median CCI of 8 . Among elderly patients, the CCI was lower in those who completed the protocol $(p=0.031)$ and those of the upfront surgery group $(p=0.006)$ (Table III).

There was no difference in terms of postoperative morbidity and mortality and surgical radicality between patients who received chemoradiation plus surgery and those who underwent upfront esophagectomy, and the CCI was similar in the two groups $(p=0.741)$. These results point out that age alone does not directly reflect a patient's ability to tolerate chemoradiotherapy or surgery, and comorbidities play a central role in the decision-making process.

The survival rates in our cohorts of patients treated with CROSS protocol or with upfront surgery were similar. Paulus et al. showed that 1-year survival was not different in octogenarians when matched with younger patients with a similar comorbidity profile. They also demonstrated that octogenarians have decreased long-term overall survival related to non-cancer-related death rate (23). Nienhueser et al. did not find any survival advantage in patients over 70 years of age who received neoadjuvant treatment (24).Therefore, the potential survival benefit of neoadjuvant treatment should probably be considered a secondary endpoint in elderly patients with multiple comorbidities.

Figure 2 shows an algorithm driven by age-adjusted CCI to be used for treatment planning in elderly patients. Based on the findings of the present study, the trimodality regimen appears prohibitive for elderly patients with a CCI score higher than 6; for these patients, palliation and supportive care may be the best choice aimed to obtain symptom relief and maintain residual quality of life. For patients with CCI of 6 or less, different therapeutic strategies should be used based on histology. Considering the reduced life expectancy, trimodality therapy is probably an overtreatment for elderly patients. For those with stage IA to IIB disease, upfront esophagectomy may be the best choice for both adenocarcinoma and squamous carcinoma. For those with stage IIIA-IIIC, histology has to be considered. Definitive chemoradiation is feasible in elderly patients (25) and is particularly effective on the squamous subtype, with salvage surgery being reserved for biopsy-positive persistent local disease $(26,27)$.

Chemotherapy guided by the molecular profile could be considered in stage IIIA-IIIC adenocarcinoma, and surgery may be performed in fit patients. Hopefully, the ESOPEC trial will provide better evidence in the future in support of perioperative chemotherapy (28). The decision-making and treatment plan in elderly patients with esophageal cancer can also be influenced by the type of surgical approach. Zahoor et al. showed that primary minimally invasive esophagectomy allows earlier return to normal function and better tolerance of adjuvant therapy (29). This approach may represent an alternative to neoadjuvant chemoradiation therapy in selected elderly patients (30).

\section{Study Limitations}

This was a retrospective, single-center study. Confounding bias due to unmeasured and unmeasurable variables cannot be excluded, nor can selection bias. Despite the sample size being limited, we were able to detect statistically significant differences in some clinically relevant parameters.

\section{Conclusion}

There is a lack of evidence regarding the outcome of trimodality therapy in elderly patients with esophageal carcinoma. Age, life expectancy, functional status, comorbidities and quality of life must be taken into account when planning oncological treatment and a tailored approach is recommended. The routine use of age-related CCI, which can be easily captured from patient's records, may provide useful information when selecting an appropriate treatment strategy for elderly patients. Minimally invasive esophagectomy represents a safe and effective approach to reducing the overall burden of treatment.

\section{Acknowledgements}

This work was supported by AIRES (Associazione Italiana Ricerca ESofago).

\section{References}

1 Bray F, Ren J-S, Masuyer E and Ferlay J: Global estimates of cancer prevalence for 27 sites in the adult population in 2008. Int J Cancer 132: 1133-1145, 2013.

2 Holmes RS and Vaughan TL: Epidemiology and pathogenesis of esophageal cancer. Semin Radiat Oncol 17: 2-9, 2007.

3 Backemar L, Lagergren P, Johar A and Lagergren J: Impact of co-morbidity on mortality after esophageal cancer surgery. Br J Surg 102: 1097-1105, 2015

4 Basta YL, Bolle S, Fockens P and Tytgat KMAJ: The value of multidisciplinary team meetings for patients with gastrointestinal malignancies: a systematic review. Ann Surg Oncol 24: 2669$2678,2017$. 
5 Pasquali S, Yim G, Vohra RS, Mocellin S, Nyanhongo D, Marriott P, Geh JI and Griffiths EA: Survival after neoadjuvant and adjuvant treatments compared to surgery alone for resectable esophageal carcinoma: a network meta-analysis. Ann Surg 265: 481-491, 2017.

6 Won E and Ilson DH: Management of localized esophageal cancer in the older patient. Oncologist 19: 367-374, 2014.

7 Edge SB, Byrd DR and Compton CC: The American Joint Committee on Cancer: the 7th edition of the AJCC Cancer Staging Manual and the future of TNM. Ann Surg Oncol 17: 1471-1474, 2010.

8 Charlson M, Szatrowski TP, Peterson J and Gold J: Validation of a combined comorbidity index. J Clin Epidemiol 47: 1245$1251,1994$.

9 Hall WH, Ramachandran R, Narayan S, Jani AB and Vijayakumar S: An electronic application for rapidly calculating Charlson comorbidity score. BMC Cancer 4: 94, 2004.

10 Bonavina L, Asti E, Sironi A, Bernardi D and Aiolfi A: Hybrid and total minimally invasive esophagectomy: how I do it. J Thorac Dis 9: S761-S772, 2017.

11 Cancer Therapy Evaluation Program. Common terminology criteria for adverse events. Version 4.0. DCTD, NCI, NIH, DHHS (http://ctep.cancer.gov), 2009.

12 Dunn OJ: Multiple comparisons using rank sums. Technometrics 6: 241-252, 1964.

$13 \mathrm{R}$ Core Team. R: A language and environment for statistical computing. R Foundation for Statistical Computing, Vienna, Austria, 2015. URL https://www.R-project.org/.

14 Scarpa M, Filip B, Cavallin F, Alfieri R, Saadeh L, Cagol M and Castoro C: Esophagectomy in elderly patients: which is the best prognostic score? Dis Esophagus 29: 589-597, 2016.

15 van Hagen P, Hulshof MC, van Lanschot JJ, Steyerberg EW, van Berge Henegouwen MI, Wijnhoven BP, Richel DJ, Nieuwenhuijzen GA, Hospers GA, Bonenkamp JJ, Cuesta MA, Blaisse RJ, Busch OR, ten Kate FJ, Creemers GJ, Punt CJ, Plukker JT, Verheul HM, Spillenaar Bilgen EJ, van Dekken H, van der Sangen MJ, Rozema T, Biermann K, Beukema JC, Piet AH, van Rij CM, Reinders JG, Tilanus HW, van der Gaast A; CROSS Group: Preoperative chemoradiotherapy for esophageal or junctional cancer. N Engl J Med 366: 2074-2084, 2012.

16 Shapiro J, van Lanschot JJB, Hulshof MCCM, van Hagen P, van Berge Henegouwen MI, Wijnhoven BPL, van Laarhoven HWM, Nieuwenhuijzen GAP, Hospers GAP, Bonenkamp JJ, Cuesta MA, Blaisse RJB, Busch ORC, Ten Kate FJW, Creemers GM, Punt CJA, Plukker JTM, Verheul HMW, Bilgen EJS, van Dekken H, van der Sangen MJC, Rozema T, Biermann K, Beukema JC, Piet AHM, van Rij CM, Reinders JG, Tilanus HW, Steyerberg EW, van der Gaast A; CROSS study group: Neoadjuvant chemoradiotherapy plus surgery versus surgery alone for oesophageal or junctional cancer (CROSS): long-term results of a randomized controlled trial. Lancet Oncol 16: 1090-1098, 2015.

17 Fogh SE, Yu A, Kubicek GJ, Scott W, Mitchell E, Rosato EL and Berger AC: Do elderly patients experience increased perioperative or postoperative morbidity or mortality when given neoadjuvant chemoradiation before esophagectomy? Int J Radiat Oncol Biol Phys 80: 1372-1376, 2011.

18 Ruol A, Portale G, Castoro C, Merigliano S, Cagol M, Cavallin F, Chiarion Sileni V, Corti L, Rampado S, Costantini M and Ancona E: Effects of neoadjuvant therapy on perioperative morbidity in elderly patients undergoing esophagectomy for esophageal cancer. Ann Surg Oncol 14: 3243-3250, 2007.
19 Camerlo A, D'Journo XB, Ouattara M, Trousse D, Doddoli C and Thomas PA: Adenocarcinoma of the esophagus and esophagogastric junction in patients older than 70 years: Results of neoadjuvant radiochemotherapy followed by transthoracic esophagectomy. J Visc Surg 149: e203-e210, 2012.

20 Bonavina L, Incarbone R, Saino G, Clesi P and Peracchia A: Clinical outcome and survival after esophagectomy for carcinoma in elderly patients. Dis Esophagus 16: 90-93, 2003.

21 Mariette C, Dahan L, Mornex F, Maillard E, Thomas PA, Meunier B, Boige V, Pezet D, Robb WB, Le Brun-Ly V and Bosset JF, Mabrut JY, Triboulet JP, Bedenne L and Seitz JF: Surgery alone versus chemoradiotherapy followed by surgery for stage I and II esophageal cancer: Final analysis of randomized controlled phase III trial FFCD 9901. J Clin Oncol 32: 24162422, 2014.

22 Klevebro F, Johnsen G, Johnson E, Viste A, Myrnäs T, Szabo E, Jacobsen AB, Friesland S, Tsai JA, Persson S, Lindblad M, Lundell L and Nilsson M: Morbidity and mortality after surgery for cancer of the oesophagus and gastro-oesophageal junction: A randomized clinical trial of neoadjuvant chemotherapy $v s$. neoadjuvant chemoradiation. Eur J Surg Oncol 41: 920-926, 2015.

23 Paulus E, Ripat C, Koshenkov V, Prescott AT, Sethi K, Stuart H, Tiesi G, Livingstone AS and Yakoub D: Esophagectomy for cancer in octogenarians: should we do it? Langenbecks Arch Surg 402: 539-545, 2017.

24 Nienhueser H, Kunzmann R, Sisic L, Blank S, Strowitzk MJ, Bruckner T, Jäger D, Weichert W, Ulrich A, Büchler MW, Ott K and Schmidt T: Surgery of gastric cancer and esophageal cancer: does age matter? J Surg Oncol 112: 387-395, 2015.

25 Münch S, Heinrich C, Habermehl D, Oechsner M, Combs SE and Duma MN: Primary radio(chemo)therapy for esophageal cancer in elderly patients: Are efficiency and toxicity comparable with younger patients? Eur J Med Res 22: 24, 2017.

26 Jamel S and Markar SR: Salvage esophagectomy: safe therapeutic strategy? J Thorac Dis 9: S799-S808, 2017.

27 Kumagai K, Mariosa D, Tsai JA, Nilsson M, Ye W, Lundell L and Rouvelas I: Systematic review and meta-analysis on the significance of salvage esophagectomy for persistent or recurrent esophageal squamous cell carcinoma after definitive chemoradiotherapy. Dis Esophagus 29: 734-739, 2016.

28 Hoeppner J, Lordick F, Brunner T, Glatz T, Bronsert P, Röthling N, Schmoor C, Lorenz D, Ell C, Hopt UT and Siewert JR: ESOPEC: prospective randomized controlled multicenter phase III trial comparing perioperative chemotherapy (FLOT protocol) to neoadjuvant chemoradiation (CROSS protocol) in patients with adenocarcinoma of the esophagus (NCT02509286). BMC Cancer 16: 503, 2016

29 Zahoor H, Luketich JD, Levy RM, Awais O, Winger DG, Gibson MK and Nason KS: A propensity-matched analysis comparing survival after primary minimally invasive esophagectomy followed by adjuvant therapy to neoadjuvant therapy for esophagogastric adenocarcinoma. J Thorac Cardiovasc Surg 149: 538-547, 2015.

30 Bonavina L, Scolari F, Aiolfi A, Bonitta G, Sironi A, Saino G and Asti E: Early outcome of thoracoscopic and hybrid esophagectomy: Propensity-matched comparative analysis. Surgery 159: 1073-1081, 2016.

Received December 29, 2017

Revised January 26, 2018

Accepted January 30, 2018 